\title{
Urothelial carcinoma associated with a blind-ending bifid ureter
}

\author{
Koichi Kodama $\cdot$ Yoichi Iwasa $\cdot$ Isamu Motoi • \\ Yasukazu Takase $\cdot$ Katsuhiko Saito
}

Received: 8 August 2011/ Accepted: 2 March 2012/Published online: 30 March 2012

(C) The Japan Society of Clinical Oncology 2012

\begin{abstract}
We report a case of urothelial carcinoma (UC) associated with a blind-ending bifid ureter. A 76-year-old man presented with asymptomatic, intermittent gross hematuria. Computed tomography revealed a mass in the left middle ureter at the level of the pelvic rim, which appeared to be the cause of hydronephrosis. A pouch of the ureter was also detected, and it was closely associated with the dilated main ureter along its entire length. Retroperitoneoscopic nephroureterectomy with open excision of the bladder cuff was performed. Pathological examination of the surgical specimen revealed two papillary UCs. The main tumor of dimension $30 \times 15 \times 15 \mathrm{~mm}$ was mainly located at the proximal end of the blind-ending branch and a solitary daughter tumor of dimension $5 \times 5 \times 5 \mathrm{~mm}$ was within the pouch. The main tumor was thought to originate from the blind-ending ureter and invade the adjacent main ureter, thereby causing the dilatations of the main and blind-ending bifid ureter. This is the second reported case of UC associated with a blind-ending bifid ureter. A longterm adverse effect of the blind-ending branch on the urothelial microenvironment may have important implications in the pathogenesis of malignancy.
\end{abstract}

Keywords Urothelial carcinoma - Ureteral tumor - Blindending bifid ureter $\cdot$ Nephroureterectomy $\cdot$ Laparoscopy

K. Kodama ( $₫)$ · Y. Iwasa · I. Motoi · Y. Takase Department of Urology, Toyama City Hospital,

2-1 Imaizumihokubu-cho, Toyama, Toyama 939-8511, Japan

e-mail: kokodama@tch.toyama.toyama.jp

K. Saito

Department of Pathology, Toyama City Hospital,

2-1 Imaizumihokubu-cho, Toyama, Toyama 939-8511, Japan

\section{Introduction}

A bifid ureter with a blind-ending branch is a rare congenital anomaly of the ureter. This anomaly is diagnosed three times more frequently in women than in men and is observed twice as often on the right side as on the left side of the ureter [1]. Most patients with a blind-ending bifid ureter remain asymptomatic throughout their lives, leading to low incidence. The two most common symptoms are recurrent urinary tract infection and poorly defined abdominal pain. Other symptoms include renal colic pains and hematuria. At the blind-ending bifid branch, urinary stasis because of disturbed peristalsis may occur, which is known to cause chronic inflammation, infection, or calculus formation [2, 3]. However, urothelial carcinoma (UC) within the blind-ending branch is extremely rare and only one case report has been published so far [4]. Here we report a case of UC associated with a blind-ending bifid ureter treated by retroperitoneoscopic nephroureterectomy.

\section{Case report}

A 76-year-old man on medication for high blood pressure presented with intermittent gross hematuria of 1-day duration. He was afebrile and denied having abdominal discomfort, flank pain, or voiding symptoms. Physical examination and blood tests were unremarkable. Medical history was not significant for malignancy, surgery, radiation exposure, or history of tobacco use. Urinalysis showed 10-19 red blood cells per high-power field and a sterile culture. The results of voiding urine cytology were UC. Cystoscopy was completely normal.

Transverse computed tomography (CT) imaging revealed a mass in the left middle ureter of size $16 \times 13 \mathrm{~mm}$ at the 

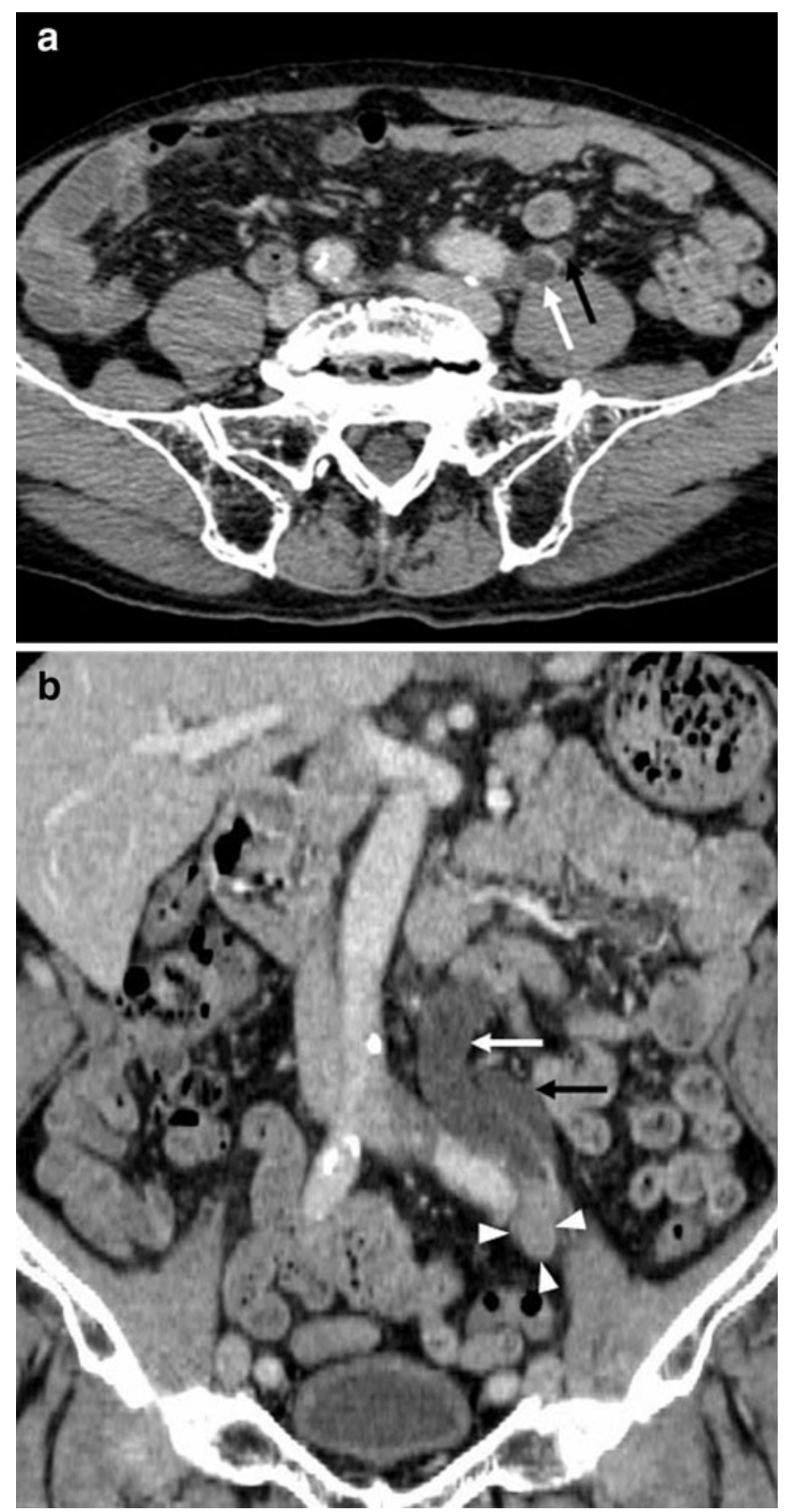

Fig. 1 Contrast-enhanced CT images of the abdomen. a Transverse CT shows a blind-ending bifid ureter (black arrow) is closely associated with the dilated main ureter (white arrow) along its entire length. b Coronal CT shows a tumor (arrowheads) in the left middle ureter at the level of the pelvic rim. The tumor is located at the bifurcation of the branch, thereby causing the dilatations of the main (white arrow) and blind-ending bifid ureter (black arrow)

level of the pelvic rim. This appeared to be the cause of hydronephrosis. A pouch of the ureter was also detected, and it was closely associated with the dilated main ureter along its entire length (Fig. 1a). Coronal enhanced CT revealed a blind-ending ureteral branch of a Y-junction joining the main ureter at an acute angle. The tumor was located at the bifurcation, thereby causing dilatations of the main ureter and the blind-ending branch (Fig. 1b). The preoperative diagnosis was a ureteral tumor staged as T2NOM0.

Laparoscopic nephroureterectomy with open excision of the bladder cuff was performed. A four-port retroperitoneal approach was employed. After dissecting the left kidney, the main ureter and blind-ending bifid branch were seen to be lying adjacent to each other within a common sheath. After careful dissection of the sheath from the surrounding tissue, the kidney was removed along with the ureter by a median incision of the lower abdomen.

The gross appearance of the surgical specimen revealed a dilated blind-ending branch measuring approximately $7 \mathrm{~cm}$ in length and a main ureter within the same sheath (Fig. 2a). A cut section of the main ureter and the blind branch revealed two papillary tumors. The main tumor of dimension $30 \times 15 \times 15 \mathrm{~mm}$ was mainly located at the proximal end of the blind-ending branch and a solitary daughter tumor of dimension $5 \times 5 \times 5 \mathrm{~mm}$ was within the pouch (Fig. 2b). Microscopic examination of the main tumor revealed UC, papillary, invasive, grade 3, INF $\beta$, pT2, u-lt0, ew0, ly1, v0 (Fig. 3). The daughter tumor was UC, papillary, non-invasive, grade 3 , pT 1 . The main tumor was thought to originate from the blind-ending ureter and invade the adjacent main ureter, thereby causing hydronephrosis. There was no evidence of nephrogenic tissue within the blind portion.

The postoperative course was uneventful. The recommended treatment was adjuvant chemotherapy, but the patient chose not to receive it. Six months later, he had a solitary papillary tumor at the posterior wall of the bladder. Transurethral resection of the bladder tumor was performed and pathological examination revealed UC, papillary, non-invasive, grade $3, \mathrm{pT} 1$. The patient has been well without any evidence of disease 6 months after transurethral surgery without adjuvant treatment.

\section{Discussion}

Blind-ending bifid ureters are a result of abnormal embryogenesis. At 4 weeks gestation, the ureteral bud spouts from the mesonephric duct and grows cranially towards the metanephric mesenchyme [5]. If two ureteral buds arise from the mesonephric duct and each bud makes contact with the metanephric mesenchyme, the result will be two separate renal units and collecting systems, and ureteral orifices. Fission of the ureteral bud with failure of one of the branches to reach or stimulate the metanephric mesenchyme results in a blind-ending bifid ureter. Histologically, the blind segment contains all normal ureteral layers.

At the blind-ending branch, urinary stasis because of disturbed peristalsis may occur. Uretero-ureteral reflux 


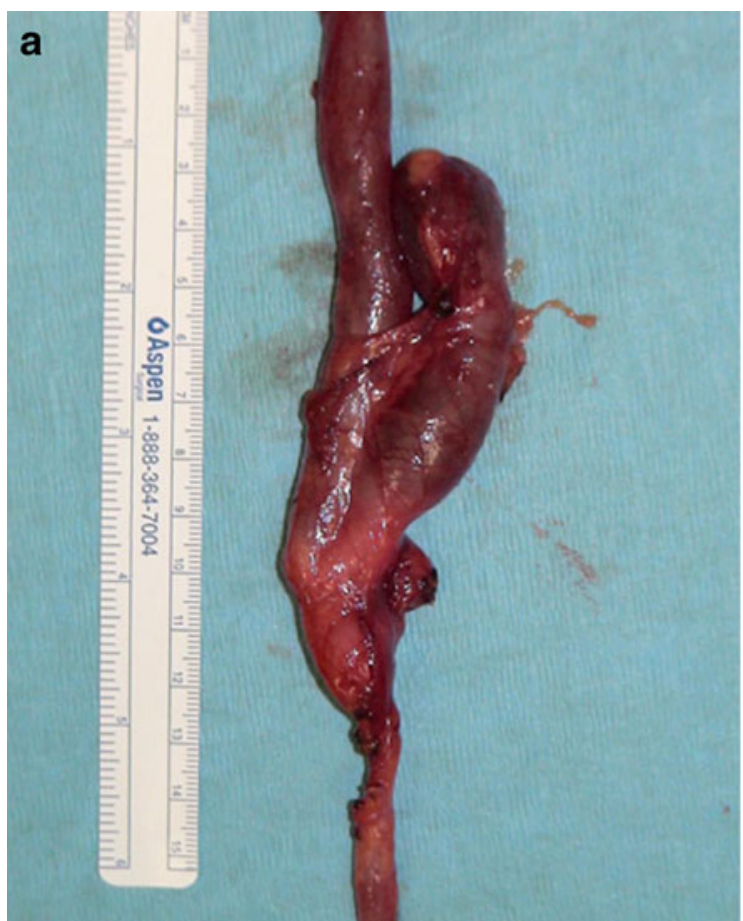

Fig. 2 Gross appearance of the surgical specimen. a A dilated blindending bifid branch and the main ureter are within the same sheath. b A cut section of the main ureter and blind branch reveals two

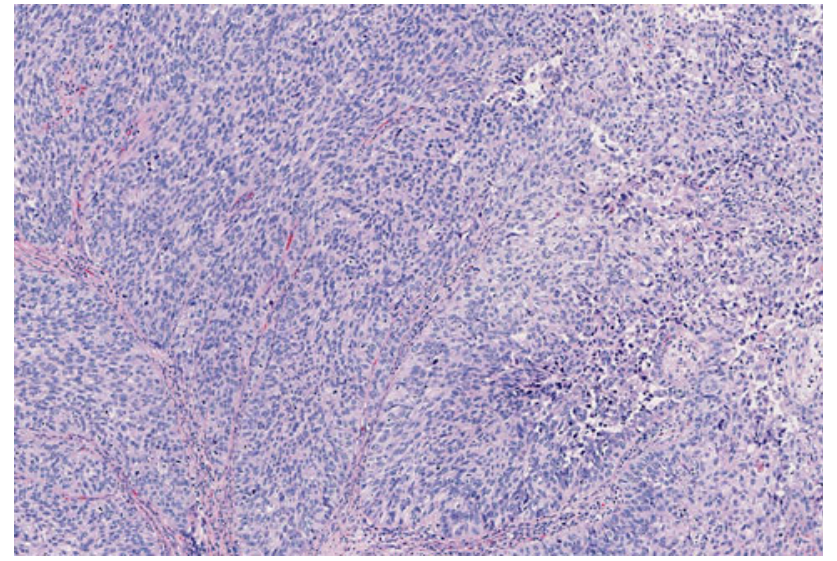

Fig. 3 Microscopic examination of the main tumor. Histological diagnosis is urothelial carcinoma, papillary, invasive, grade 3 . H\&E staining, $\times 100$

from the main ureter into the blind-ending branch as a result of peristaltic waves of the two limbs has been demonstrated by cineradiography [6] and multi-detector CT [7]. In particular, antegrade peristalsis occurs in one branch and, when it reaches the bifurcation, it proceeds into the other branch as a retrograde wave. The two branches tend to alternate the direction of the peristaltic flow.

Chronic urinary stasis causes irritation to the urothelium leading to dysplasia, which at a later date develops into an overt cancer. Bladder diverticula are outpouchings of the urothelial lining not having the muscular layer, resulting in

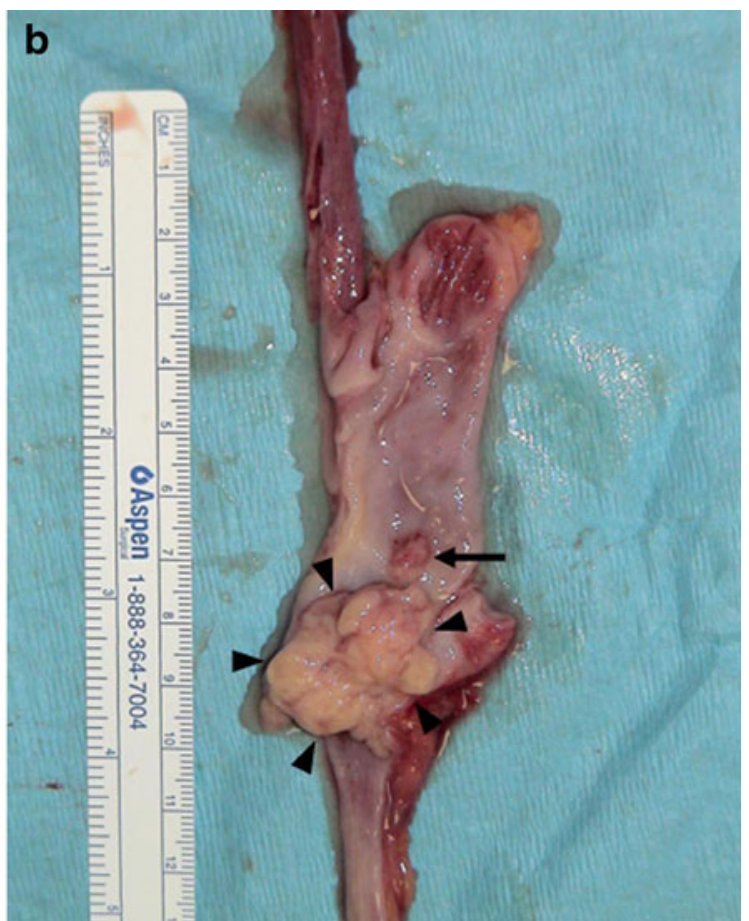

papillary tumors. The main tumor (arrowheads) is mainly located at the proximal end of the blind-ending branch and a daughter tumor (arrow) is within the pouch

loss of its contractile activity which will lead to urinary stasis. Tamas et al. [8] reported that approximately half of bladder diverticula demonstrated neoplastic findings ranging from in situ lesions to invasive carcinoma. To explain the mechanism of carcinogenesis of the urothelium, several theories have been proposed including chronic inflammation, infection, production of $N$-nitroso compounds by bacteria, and/or a change in mucosal electrolyte composition and/or $\mathrm{pH}$ [9]. A long-term adverse effect of the blind-ending branch on the urothelial microenvironment may have important implications in the pathogenesis of malignancy. Therefore, this should be investigated further.

UCs of the upper urinary tract and bladder are often multifocal and commonly develop multiple recurrences after resection. Two possible mechanisms could explain the multifocal nature and multiple recurrences of UC over time. The field effect theory argues for a polyclonal origin of multiple tumors [10]. In contrast, the implantation theory argues for a monoclonal origin of multiple urothelial tumors, suggesting that recurrences result from intraluminal seeding or intraepithelial spread of the original cancer cells [11]. Most molecular studies have favored the monoclonal origin of multiple synchronous or metachronous UCs. In the present case, uretero-ureteral reflux may have contributed to intraluminal seeding of UC inside the blind-ending branch. The reported recurrence rate within the bladder after treatment of a primary UC of the upper 
urinary tract varies considerably from 15 to $50 \%[12,13]$. Although multifocality and pathologic stage of UC of the upper urinary tract are known independent factors for bladder recurrences, in at least one study it was reported that high-grade tumors of the upper urinary tract are also associated with a greater incidence of bladder recurrence [14]. In the present case, strict follow-up after radical nephroureterectomy is mandatory to detect metachronous bladder tumors.

Careful dissection is a notable parameter because main and blind-ending ureters are present in the same sheath. For the present case with UCs inside the blind branch, extreme care was taken to avoid inadvertent rupture of the blind-ending branch and spillage of tumor cells during excision. The sheath is less dense at the upper or proximal end. It has been reported that laparoscopic resection of the blind-ending branch in symptomatic patients has several benefits including shorter hospital stay, lesser postoperative pain, and quicker return to physical activity compared with open surgery [2]. In our experience, the laparoscopic approach has additional benefits in readily identifying the blind-ending branch and ensures its safe dissection.

In summary, we presented a case of UC associated with a blind-ending bifid ureter treated by retroperitoneoscopic nephroureterectomy. At the blind-ending branch, prolonged contact with urinary carcinogens caused by urinary stasis may occur. A long-term adverse effect of the blindending branch on the urothelial microenvironment may have important implications in the pathogenesis of malignancy.

Conflict of interest None.

\section{References}

1. Albers DD, Geyer JR, Barnes SD (1971) Clinical significance of the blind-ending branch of a bifid ureter: report of 3 additional cases. J Urol 105:634-637

2. Perlmutter AE, Parousis VX, Farivar-Mohseni H (2005) Laparoscopic retroperitoneal resection of blind-ending bifid ureter in patient with recurrent urinary tract infections. Urology 65:388

3. Halim HA, Al-Awadi KA, Kehinde EO et al (2005) Blind-ending ureteral duplication with calculi. Ann Saudi Med 25:346-348

4. Kawamura H, Sasaki N (1998) Transitional cell carcinoma in the blind-ending branch of the bifid ureter. Br J Urol 82:307-308

5. Peterson LJ, Grimes JH, Weinerth JL et al (1975) Blind-ending branches of bifid ureters. Urology 5:191-195

6. Lenaghan D (1962) Bifid ureter in children: an anatomical, physiological and clinical study. J Urol 87:808-817

7. Chang E, Santillan C, O'Boyle MK (2011) Blind-ending branch of a bifid ureter: multidetector CT imaging findings. Br J Radiol 84:e38-e40

8. Tamas EF, Stephenson AJ, Campbell SC et al (2009) Histopathologic features and clinical outcomes in 71 cases of bladder diverticula. Arch Pathol Lab Med 133:791-796

9. Gitlin JS, Wu XR, Sun TT et al (1999) New concepts of histological changes in experimental augmentation cystoplasty: insights into the development of neoplastic transformation at the enterovesical and gastrovesical anastomosis. J Urol 162:1096-1100

10. Paiss T, Wöhr G, Hautmann RE et al (2002) Some tumors of the bladder are polyclonal in origin. J Urol 167:718-723

11. Sidransky D, Frost P, Von Eschenbach A et al (1992) Clonal origin bladder cancer. N Engl J Med 326:737-740

12. Raman JD, Ng CK, Boorjian SA et al (2005) Bladder cancer after managing upper urinary tract transitional cell carcinoma: predictive factors and pathology. BJU Int 96:1031-1035

13. Terakawa T, Miyake H, Muramaki M et al (2008) Risk factors for intravesical recurrence after surgical management of transitional cell carcinoma of the upper urinary tract. Urology 71:123-127

14. Oehlschläger S, Baldauf A, Wiessner D et al (2004) Bladder tumor recurrence after primary surgery for transitional cell carcinoma of the upper urinary tract. Urol Int 73:209-211 